\title{
Fatty Acid-Binding Protein 4 in Endometrial Epithelium Is Involved in Embryonic Implantation
}

\author{
Peng Wang ${ }^{\mathrm{a}}$ Qiuyuan Zhu ${ }^{\mathrm{a}}$ Huilian Peng ${ }^{\mathrm{a}}$ Mengkai Du ${ }^{\mathrm{a}}$ Hanzhi Wang ${ }^{\mathrm{a}}$ \\ Minyue Dong $g^{\mathrm{a}, \mathrm{b}, \mathrm{c}}$
}

aWomen's Hospital, School of Medicine, Zhejiang University, Hangzhou, 'Key Laboratory of Women's Reproductive Health of Zhejiang Province, Hangzhou, 'Key Laboratory of Reproductive Genetics of the Ministry of Education, Hangzhou, China

\section{Key Words}

Fatty acid binding protein 4 - Endometrium - Embryonic implantation - Pregnancy loss • Receptivity

\begin{abstract}
Aims: To clarify the role of fatty acid-binding protein 4 (FABP4) of endometrial epithelial cell in the establishment and maintenance of pregnancy and the involvement in the pathogenesis of pregnancy loss. Methods: The expression of FABP4 and uterine receptive factor (LIF, Integrin- $\beta 3$ and Claudin 4) was determined by Western blotting or quantitative PCR. FABP4 siRNA was used to silence FABP4 while FABP4 inhibitor was used to inhibit the function of FABP4 in endometrial epithelial cell. ICR mice were raised to evaluate the effect of FABP4 silence or inhibition on embryo implantation in vivo after FABP4 siRNA mixture or inhibitor was injected into uterus, and an embryonic adhesion system using trophoblast spheroids mimicking embryos was set up to assess the effect of FABP4 silence or inhibition on embryonic adhesion onto endometrial cell in vitro. Results: The expression of FABP4 mRNA was significantly decreased in the deciduas of women with pregnancy loss compared with that of women with normal pregnancy. FABP4 siRNA significantly reduced the number of embryos implanted and FABP4 expression in ICR mice. FABP4 inhibition also significantly decreased the number of embryos implanted. Either silence or inhibition of FABP4 in endometrial epithelial cell abolished the expression of uterine receptive factors induced by the combination of estrogen and progesterone-induced, and reduced the number of trophoblast spheroids adhered onto endometrial cell. Conclusions: FABP4 regulates embryo implantation via altering uterine receptivity and decreased expression of FABP4 in endometrium may be linked with pregnancy loss, indicating FABP4 has biological role in the establishment and maintenance of pregnancy and subsequently is involved in pathogenesis of pregnancy loss.




\section{Cellular Physiology Cell Physiol Biochem 2017;41:501-509 and BiOChemistry Published onIIne: January 30, 2017 \begin{tabular}{l|l} 
DOI: 10.1159/000456886 2017 The Author(s). Published by S. Karger AG, Basel \\
www.karger.com/cpb
\end{tabular}}

Wang et al.: FABP4 and Embryonic Implantation

\section{Introduction}

The establishment and maintenance of pregnancy occurs through the interaction between maternal uterine endometrium and embryo (mainly the trophoblasts) [1-4]. Pathologies lead to pregnancy loss must ultimately, either directly or indirectly, affect this interaction; vice versa, abnormalities affecting this interaction will result in pregnancy loss [3-11]. The interaction between uterine endometrium and embryo is a complex process, and the molecular mechanism remains largely unknown.

Fatty acid-binding protein (FABP) 4 is a member of FABP family of highly homologous cytosolic protein binding a variety of hydrophobic ligands, such as long-chain fatty acid, encosanoids, leukotrienes, and postglandins, and is critical for some cellular processes including uptake and trafficking of fatty acid, regulation of gene expression [12,13]. Tian et al. [14] found that FABP4 was not detectable in mouse uterus from days 1 to 4 of pregnancy, appeared in primary decidual zone on days 5 and 6, and markedly up-regulated on days 7 and 8. They further confirmed FABP4 is essential for the decidualization in mouse [14]. We [15] and others [14] observed that FABP4 mRNA expression is higher in human endometrium on day LH+7 than that on day LH+2, and further, FABP4 expression was up-regulated by estrogen and further up-regulated by the combination of estrogen and progesterone. In previous investigation, we reveled with immunohistochemistry that FABP4 is constitutively expressed in the epithelial cells of endometrium at proliferative and secretory phases while FABP4 is positive in stromal cells at secretory phase [15]. Using the FABP4-expressing endometrial cells Ishikawa (a cell line derived from endometrial carcinoma) and RL952 (a cell line derived from normal endometrial epithelium), we found FABP4 is an important regulator for the proliferation, migration and invasion of endometrial epithelial cells [15]. These findings point to the importance of FABP4 in the biology of uterine endometrium. However, it remains unknown whether FABP4 is involved in the process of pregnancy loss and the interaction between embryo and uterine endometrium.

In vitro and in vivo models have been established to evaluate embryonic adhesion and implantation. Because of the ethical and legal restriction using human embryo and because of limited access to primary endometrial cell, human endometrial and trophoblast cell lines are used in implantation studies. In the in vitro system, trophoblast mimicking embryo is cultured on monolayer endometrial cell mimicking uterine endometrium. For in vivo study, mice are often used and uterine horn injection is the route for the administration of factor studying. The effect of neutralizing antibody, specific inhibitor, siRNA, or bioactive peptide on embryonic adhesion and implantation may be observed in in vitro and in vivo models.

We hypothesized FABP4 may play a role in the establishment and maintenance of pregnancy, one of the most important biological functions of human uterine endometrium. To verify our hypothesis, we first determined alteration in FABP4 expression in human deciduas from women with pregnancy loss, and observed the effect of FABP4 on embryonic implantation in vivo and in vitro, and then explored the regulatory effect of FABP4 on endometrial receptivity.

\section{Materials and Methods}

Case control study

Women with early pregnancy loss (EPL, $n=15)$ and age-matched normal pregnant women $(n=15)$ undergoing terminations of pregnancy for social reasons designated as the control were recruited with the approval of the Institutional Review Board, Women's Hospital, School of Medicine, Zhejiang University. All participants gave their informed consent.

Pregnancy was diagnosed upon positive human chorionic gonadotropin (hCG) test after missed menstruation and confirmed by transvaginal ultrasound. Early pregnancy loss was defined as spontaneous loss before 12 completed weeks of pregnancy with no or minimal vaginal bleeding, and was documented as the absence of fetal viability that was confirmed by ultrasonography [9]. Women with a history of recurrent 


\section{Cellular Physiology Cell Physiol Biochem 2017;41:501-509

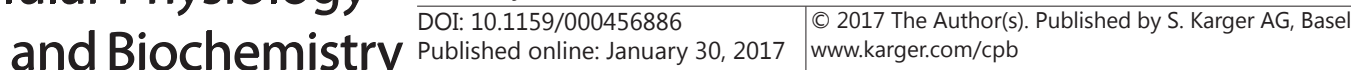

Wang et al.: FABP4 and Embryonic Implantation

spontaneous abortions, any other gynecological diseases, any other medical complications, or any chemical agent intake before terminations were excluded. Decidual tissues were stored at $-80^{\circ} \mathrm{C}$ for FABP 4 analysis.

Real-time PCR was used to detect mRNA expression of FABP4 in human decidual tissues, and glyceraldehydes-3-phosphate dehydrogenase (GAPDH) was used as the internal control. Total RNA was extracted with Trizol reagent (Invitrogen, Carlsbad, CA, USA) and reversely transcribed with PrimeScript RT reagent (TakaRa, Tokyo, Japan). Real-time PCR was conducted in duplicate using SYBR Premix EX Taq on ABI PRISM 7900 (Perkin-Elmer, Foster city, CA, USA). The sequences of primers were as following:

FABP4 forward: 5'-GCCAGGAATTTGACGAAG-3',

reverse: 5'-ATCCCACAGAATGTTGTAGAGT-3';

GAPDH forward: 5'-CAGGGCTGCTTTTAACTCTGG-3',

reverse: 5'-TGGGTGGAATCATATTGGAACA-3';

The length of PCR products was $256 \mathrm{bp}$ and $102 \mathrm{bp}$ for FABP4 and GAPDH respectively. The cycling conditions were as follows: $95^{\circ} \mathrm{C}$ for $10 \mathrm{~s}$, and then 40 cycles of $95^{\circ} \mathrm{C}$ for $5 \mathrm{~s}$ and $60^{\circ} \mathrm{C}$ for $30 \mathrm{~s}$. The relative expression of target genes was calculated with comparative threshold (CT) method using following formula:

Relative expression $=2^{-\Delta \Delta \mathrm{CT}}$

where, $\Delta \Delta \mathrm{CT}=\Delta \mathrm{CT}$ (early pregnancy loss) $-\Delta \mathrm{CT}$ (normal pregnancy), and $\Delta \mathrm{CT}=\mathrm{CT}$ (FABP4) $-\mathrm{CT}$ (GAPDH).

\section{Animal model}

ICR mice of 8-10 weeks were caged in a temperature- and humidity-controlled environment with a 12-hour light/12-hour dark cycle in the First Affiliated Hospital, School of Medicine, Zhejiang University. Animals were cared according to the protocol approved by the Institutional Animal Care and Use Committee of Zhejiang University. Female mice were caged with male mice at the ratio of 2 to 1 . On the second day, vaginal plug was examined. When vaginal plug was observed, the day was designated day 1 of pregnancy. To observe the effect of FABP4 siRNA (small interference of RNA) on embryonic implantation in vivo, a mixture of FABP4 siRNA (Santa Cruz Inc., CA, USA; catalog number: sc-43592; 10 $\mu \mathrm{M}, 15 \mu \mathrm{L}$ ) was injected into one of the uterine horns and scrambled siRNA (Santa Cruz; sc37007; 10nM, 15uL) into the other side on day 3 after anesthetized with intraperitioneal injections of $4 \%$ chloral hydrate $(0.1 \mathrm{ml} / 10 \mathrm{~g})$. Uteri were collected for western blotting analysis on day 4 or for embryo counting on day 7 after killed with cervical dislocation. To determine the effect of specific FABP4 inhibitor, FABP4 inhibitor $(100 \mu \mathrm{M}, 15 \mu \mathrm{L})$, which is cell-permeable and targets the fatty acid-binding pocket, was injected into one of uterine horn and the vehicle into the other side on day 3 after anesthetized the same as the description before, and embryos were counted on day 7 after killed with cervical dislocation.

Tissues were lyzed in $50 \mu \mathrm{l}$ protein lysis buffer containing protease inhibitors. BCA Protein Kit (Applygen Inc. Ltd, Beijing, China) were used to determine protein concentration. Proteins were separated on $12 \%$ sodium dodeeylsulfate- polyacrylamine gel electrophoresis (SDS-PAGE) and transferred onto polyvinylidene difluoride (PVDF) membranes (Millipore, Massachusetts, USA). The membranes were blocked with Tris-buffered saline (TBS) and $0.1 \%$ Tween 20 (TBS/T) containing 5\% bovine serum albumin and then incubated with specific primary antibodies (Anti-FABP4 antibody: Cell Signaling Technology, USA; diluted at 1:800; Anti-glyceraldehyde-3-phosphate dehydrogenase (GAPDH) antibody: Cell Signaling Technology Co; 1:1000) diluted in TBS/T at room temperature for 1 hour or at $4{ }^{\circ} \mathrm{C}$ overnight. The membranes were washed three times with TBS/T and then incubated with the appropriate HRP-conjugated secondary antibodies (MultiSciences(Lianke) Biotech Co., Ltd. China, Hangzhou) for 1 hour at room temperature. The membranes were washed three times with TBS/T. The bands were detected by enhanced-chemiluminescence and visualized by autoradiography. The relative expression of FABP4 was normalized against internal control GAPDH respectively.

\section{In vitro embryonic adhesion analysis}

Cells were cultured as previously described [15]. Briefly, Ishikawa and Jar cells were cultured in RPMI1640 medium (phenol red free) while RL952 cell in Dulbecco's Modified Eagle Media: Nutrient Mixture F-12 (F12/DMEM 1:1; phenol red free) supplemented with 10\% fetal bovine serum (FBS) and 1\% penicillin/ streptomycin at $37^{\circ} \mathrm{C}$ in a humidified atmosphere of $5 \% \mathrm{CO}_{2} .17-\beta$ estradiol, $10^{-8} \mathrm{~mol} / \mathrm{l}$, and progesterone $\left(10^{-6} \mathrm{~mol} / \mathrm{l}\right)$ were used to stimulate the expression of leukemia inhibitory factor (LIF), Integrin $\beta 3$ (ITGB3) and Claudin 4 (CLDN4) in endometrial cells. 
Cell was transfected with FABP4 siRNA (Santa Cruz Inc., CA, USA; catalog number: sc-43592; concentration: $10 \mu \mathrm{mol} / \mathrm{mL}$ ) or scrambled siRNA (Santa Cruz; sc37007) using Lipofectamine 2000 (Life Technologies Co., Taipei, Taiwan) according to the manufacturer's protocols. The transfection medium was replaced with complete medium $6 \mathrm{~h}$ after transfection, and the cells were incubated for further experiment. To inhibit the function of FABP4, a selective inhibitor of FABP4 (EMD Millipore Inc., Darmstadt, Germany; catalog number: 341310), ((2'-(5-Ethyl -3,4-diphenyl-1 H-pyrazol-1-yl) (1,1'-biphenyl)-3-yl)oxy)-acetic acid, which targeted fatty acid binding pocket, was added to the medium at the indicated concentrations for 12 hours and then the medium was replaced.

Cells was lyzed for Western blotting as described above. Primary antibodies used were as flows: Anti-FABP4 antibody: Cell Signaling Technology, USA; diluted at 1:800; anti-LIF antibody: Abcam, USA; 1:1000; anti-integrin $\beta 3$ antibody: Abcam; 1:1000; anti-Claudin 4 antibody: Santa Cruz, USA; 1:1000; Antiglyceraldehyde-3-phosphate dehydrogenase (GAPDH) antibody: Cell Signaling Technology Co; 1:1000.

Trophoblast spheroids were generated by rotating the trypsinized Jar cells at $110 \mathrm{rpm}$ for $72 \mathrm{~h}$. Ishikawal cells $\left(1 \times 10^{5}\right)$ were cultured in each well in triplicates in 12-well plate culture dishes and incubated at $37^{\circ} \mathrm{C}$. After cells covered about $50 \%$, FABP4 siRNA or inhibitor was administrated to knock down FABP4 or to inhibit the function of FABP4 as described above. The spheroids were transferred onto the surface of a confluent monolayer of an endometrial Ishikawa cell line and incubated at $37^{\circ} \mathrm{C}$ for 1 hour. The incubation dishes were inverted and centrifuged at $10 \mathrm{~g}$ for 10 minutes to remove the non-adherent spheroids. Attached spheroids were counted under a light microscope, and expressed as the percentage of the total number of spheroids used.

\section{Statistical analysis}

The Kolmogorov-Smirnov test was used to evaluate the distribution of data. The data were presented in means \pm SD of at least three separate experiments. Student t-test and one-way analysis of variance (ANOVA) followed by a Dunnett's post-hoc test were used for the comparison of normally distributed data. SPSS statistical package (Statistical Analysis System, Chicago, IL, USA) was used for the statistic analysis. Values of $\mathrm{P}<0.05$ were considered significant.

\section{Results}

Case control study

The maternal age ranged from 20 to $35(28.53 \pm 5.24)$ years for EPL women and from 20 to $40(26.53 \pm 5.37)$ years for control, respectively. The gestational age ranged from 47 to $63(54.80 \pm 4.14)$ days for EPL women and from 50 to 66 days $(56.53 \pm 5.93 \mathrm{~d})$ for control, respectively. There were no significant differences in either the maternal age $(\mathrm{P}=0.311)$ or the gestational age $(\mathrm{P}=0.057)$. Figure 1 showed FABP4 mRNA expression was significantly decreased in the deciduas of women with early pregnancy loss (EPL) compared with that of women with normal pregnancy (control) $(\mathrm{P}=0.04)$.

\section{Animal model}

As shown in Figure 2, the number of embryo implanted in the uterine horn injected with FABP4 siRNA mixture was significantly less than that in uterine horn injected with

Fig. 1. Comparison of FABP4 expression in deciduas. The expression of FABP4 mRNA was determined by real time PCR. FABP4 expression was significantly lower in early pregnancy loss than control.

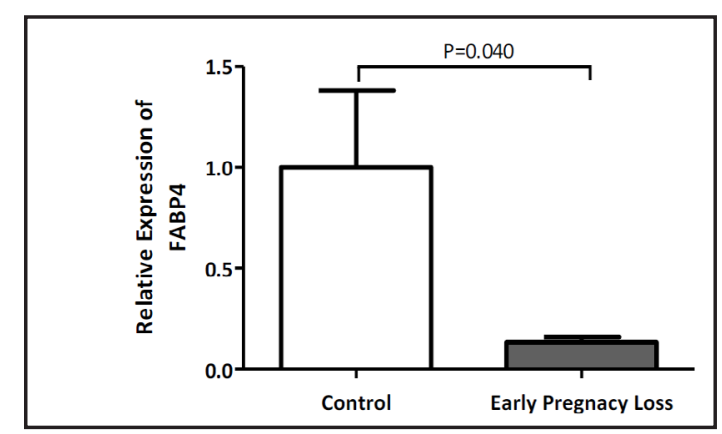


Fig. 2. The effect of FABP4 on embryo implantation in vivo. FABP4 siRNA or inhibitor was injected into one uterine horn of ICR mice, and scramble siRNA or vehicle in the opposite on Day 3. FABP4 mRNA was determined with real time PCR on Day 4 and the number of embryo implanted was counted on Day 7. A: FABP4 siRNA decreased the number of embryo implanted compared with control and the expression of FABP4. B: FABP4 inhibitor reduced the number of embryo implanted.
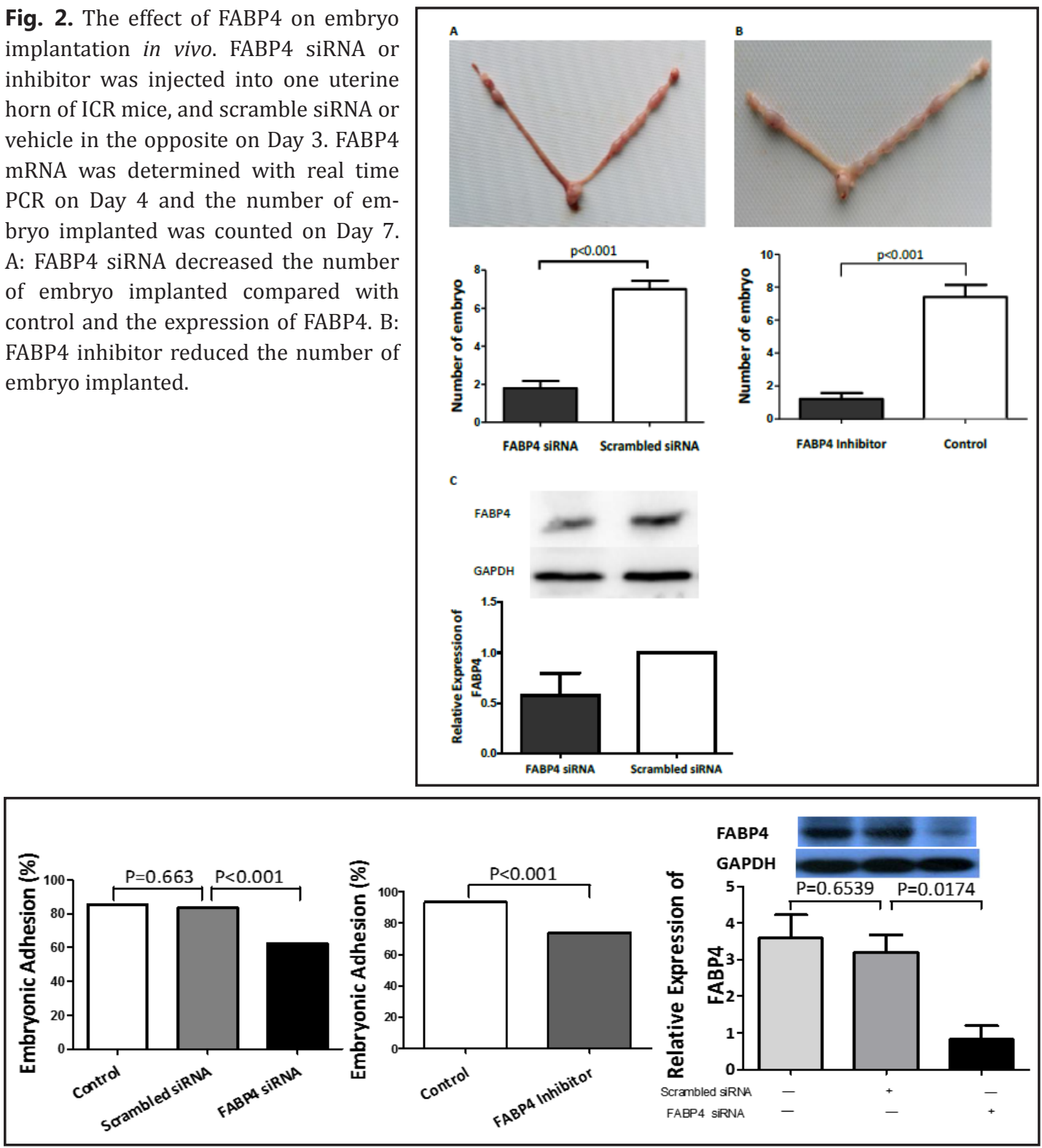

Fig. 3. The effect of FABP4 on embryo adhesion in vitro. FABP4 siRNA or inhibitor was used to inhibit FABP4 in endometrial epithelial cell and the adhesion of trophoblast spheroids onto endometrial epithelium was observed. FABP4 expression in endometrial epithelium was determined by Western blotting. A: FABP4 siRNA significantly reduced the number of trophoblast spheroid adhered. B: FABP4 inhibition significantly decreased the number of trophoblast spheroid adhered. C: FABP4 siRNA significantly decreased FABP4 expression.

scrambled siRNA (1.2 \pm 0.84 versus $7.4 \pm 1.67 ; \mathrm{P}<0.001)$. Meanwhile, western blot analysis revealed FABP4 siRNA reduced the expression of FABP4. The injection of FABP4 inhibitor into uterine horn significantly reduced the number of embryos implanted as compared with the other side (control horn) $(1.80 \pm 0.84$ versus $7.00 \pm 1.00 ; \mathrm{P}<0.001)$.

In vitro embryonic adhesion analysis

Figure 3 showed that FABP4 silence in endometrial cell line significantly reduced the number of trophoblast spheroid adhered onto endometrial cell compared with scramble 


\section{Cellular Physiology Cell Physiol Biochem 2017;41:501-509

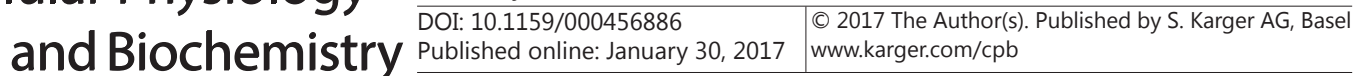

Fig. 4. The regulation of endometrial receptivity by FABP4. Estrogen and progesterone were used to induce FABP4 in endometrial epithelial cells and FABP4 miRNA or inhibitor was used to inhibit FABP4. The expression of FABP4, LIF, Integrin- $\beta 3$ and Claudin 4 was determined by Western blotting. A, C, D, F, H: Estrogen and progesterone induced FABP4 expression while FABP4 siRNA inhibited the expression of FABP4 (A and C), LIF (A and $D$ ), Integrin- $\beta 3$ (A and F) and Claudin 4 (A and $\mathrm{H}$ ). B, E, G, I: FABP4 inhibitor reduced the expression of LIF (B and E), Integrin- $\beta 3$ (B and $G$ ) and Claudin 4 (B and I).

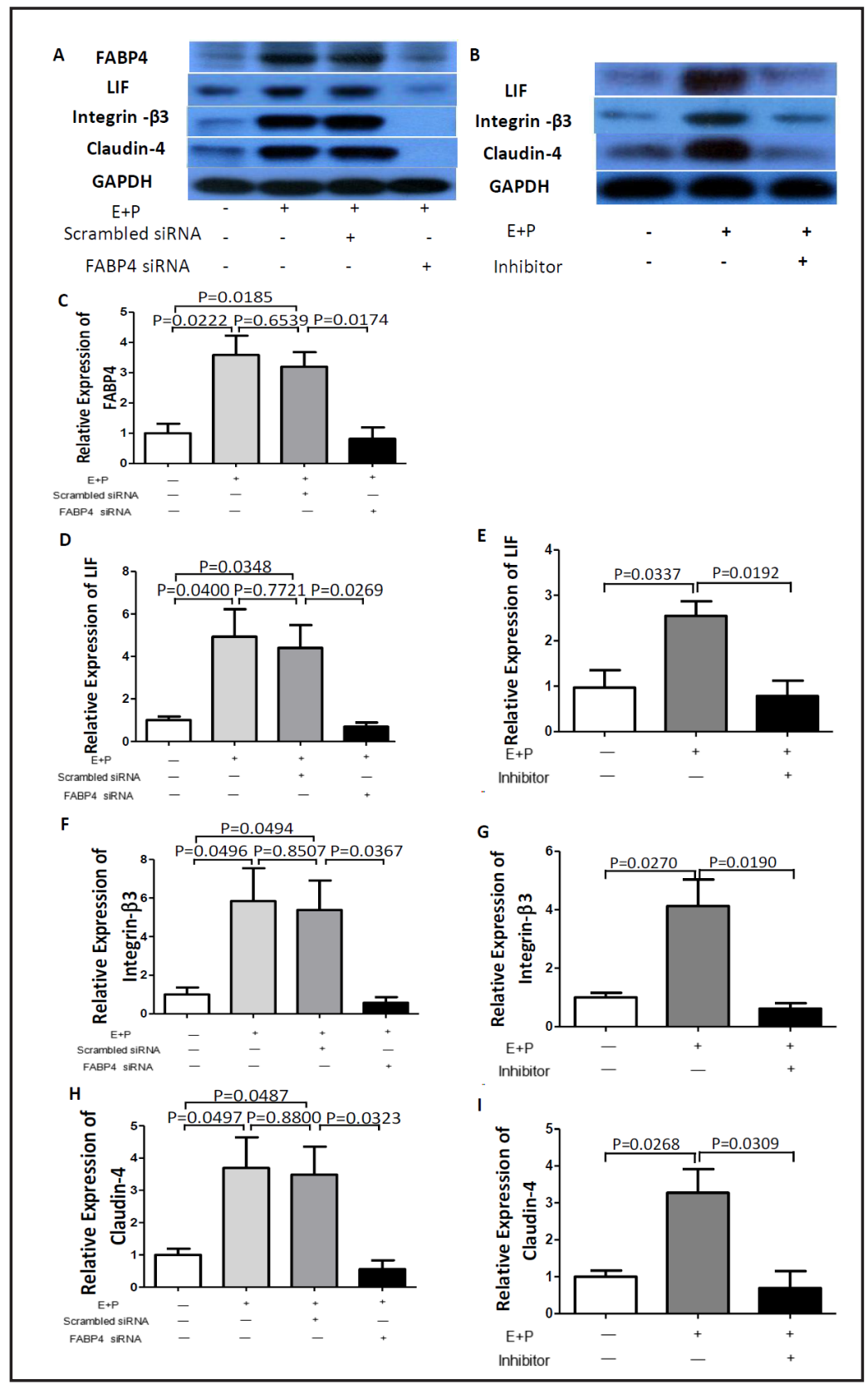

$(\mathrm{P}<0.001)$, meanwhile FABP4 siRNA significantly inhibited FABP4 expression $(\mathrm{P}=0.0174)$. Figure 3 also showed that the inhibition of FABP4 with specific inhibitor in endometrial cell significantly decreased the number of embryo adhered onto the endometrial cell as compared with control $(\mathrm{P}<0.001)$.

Uterine receptivity is the critical factor affecting embryonic implantation. To elucidate whether FABP4 affects embryonic implantation via regulating endometrial receptivity, we observed the effect of FABP4 on endometrial receptivity factors LIF, Integrin- $\beta 3$ and Claudin 4 in endometrial cell lines Ishikawa and RL952. As shown in Figure 4, the combination of estrogen and progesterone $(\mathrm{E}+\mathrm{P})$ significantly up-regulated the expression of FABP4 $(\mathrm{P}<0.05)$, LIF $(\mathrm{P}<0.05)$, Integrin- $\beta 3(\mathrm{P}<0.05)$ and Claudin $4(\mathrm{P}<0.05)$. FABP4 siRNA abolished $\mathrm{E}+\mathrm{P}$-induced expression of FABP4 ( $\mathrm{P}=0.0174)$, LIF $(\mathrm{P}=0.0269)$, Integrin- $\beta 3(\mathrm{P}=0.0367)$ and Claudin $4(\mathrm{P}=0.0323)$ in endometrial cell line. And, we found that FABP4 inhibitor also reduced $\mathrm{E}+\mathrm{P}$-induced expression of LIF $(\mathrm{P}=0.0192)$, Integrin- $\beta 3(\mathrm{P}=0.0190)$ and Claudin 4 $(\mathrm{P}=0.0309)$, in endometrial cells. 


\section{Discussion}

FABP4 is expressed in a broad spectrum of tissues and regulates a series of cellular functions including proliferation and survival, migration, and invasion [12, 15]. Previously, we observed FABP4 is constitutively expressed in endometrial epithelial cell and regulate the proliferation and survival, migration and invasion of endometrial epithelial cell. This expression of FABP4 is enhanced by estrogen and further enhanced by the combination of estrogen and progesterone $[14,15]$. In the current investigation, we have shown, for the first time, that FABP4 expressed in endometrial cell is involved in embryonic implantation via regulating uterine receptivity and that FABP4 play a role in the establishment and maintenance of pregnancy. Our findings add new insight into the function of FABP4 in female reproduction.

Pregnancy begins with the interaction between competent blastocyst and receptive endometrium, and is maintained by adequate endometrial response to the invading embryo $[1,2,4]$. Pregnancy loss is a multi-factorial disease, and endometrial abnormality is one of the most important etiologies for pregnancy loss $[3,5,9]$. The proliferation and differentiation of endometrial cells are precisely regulated by the specific genes as well as cytokines, chemokines and growth factors [6]. Recently, Manohar et al. [16] detected the expression of Sorcin, Cofilin-1, Apo-A1 and Ran, during mid-secretory phase of human endometrium, and proposed these genes may have physiological significance and their de-regulation may be one of the causes for altered differentiation and/or maturation of endometrium in women with unexplained infertility. MSX homeobox genes in uterus regulate embryonic implantation by altering uterine receptivity and paracrine signaling between epithelium and uterine stroma $[17,18]$. De-regulated genes including Prokineticin 1 were identified in the endometrium of women with idiopathic recurrent pregnancy loss [7, 19]. A series of genes, including S100A8, S100A9, selectins, and interleukin-22, were found to be differentially expressed in human deciduas from women with pregnancy loss [8,20-22]. Herein, we report significantly reduced expression of FABP4 in the deciduas of women with pregnancy loss, and we also revealed the inhibition or silence of FABP4 in mouse uterus significantly increased the embryo absorption. These findings point to the importance of FABP4 in maintenance of pregnancy.

Human pregnancy begins with the interstitial implantation of conceptus [1, 2, 4]. The initial embryonic attachment to endometrial epithelial cell takes place between the uterine gland openings $[1,2,4]$. Embryonic implantation is the first step of pregnancy and endometrial epithelial cell is the maternal cell that first meets the competent embryo [1, 2, 4]. By using in vitro model of embryo-endometrial cell interaction, we have shown the deregulation of FABP4 in endometrial cell affects embryonic attachment, and further, we revealed with in vitro experiment FABP4 regulates the receptivity of endometrial epithelium. This may be one of the mechanisms by which FABP4 participate the establishment and maintenance of pregnancy and subsequently the pathogenesis of pregnancy loss.

Endometrial epithelial cell is the maternal cell that embryo first attaches $[1,2,4]$. Endometrial epithelium may have an important biological role in governing blastocyst implantation and decidualization via secretions $[1,23]$. The secretions from endometrial gland include amino acid, carbohydrates (glucose), ions, lipids and proteins (cytokines, enzymes, hormones, growth factors, and others), and are presumed to be important mediators of uterine receptivity, blastocyt implantation (trophoblast attach, growth, and invasion), stromal cell decidualization, and embryo growth [23]. Considering that FABP4 functions as a fatty acid transporter and its expression in endometrium is elevated in midsecretory phase and could be induced by the presence of estrogen and progesterone in vitro, FABP4 may have an important role in embryonic implantation and maintenance of pregnancy, and its de-regulation may result in pregnancy loss.

The cellular localization of FABP4 in endometrium has been reported to be different in mice and humans $[14,15]$. We described previously FABP4 is present in epithelial cell of proliferative phase and in epithelial and stromal cells of mid-secretory phase. Tian et al. [14] 
observed FABP4 is expressed in stromal cell and is involved in the decidualization in mice. Both above-mentioned observations revealed FABP4 expression is regulated by estrogen and progesterone, however, it is unknown if FABP4 in human epithelial cell regulates stromal decidualization via paracrine signaling. The findings in this investigation in combination with previous reports indicate FABP4 is involved in the establishment and maintenance of pregnancy; however, much detailed mechanisms need to be investigated. An animal model with conditional deletion of FABP4 in uterus could provide much clue.

The limitations of this study include relative small size of women with pregnancy loss was recruited and embryonic aneuploidy was not excluded in women with EPL. However, these did not affect the findings from in vitro experiments and animal studies.

In summary, the expression of FABP4 is markedly decreased in deciduas of pregnancy loss and FABP 4 regulates embryo implantation via altering uterine receptivity. It is indicated FABP4 has biological roles in the establishment and maintenance of pregnancy and subsequently is involved in pathogenesis of pregnancy loss.

\section{Acknowledgements}

This work was supported by Natural Scientific Foundation of China (81170572 and 81370726).

\section{Disclosure Statement}

Authors declare they have no conflict of interest.

\section{References}

1 Spencer TE: Biological roles of uterine glands in pregnancy. Semin Reprod Med 2014;32:346-357.

2 Sharkey AM, Macklon NS: The science of implantation emerges blinking into the light. Reprod Biomed Online 2013;27:453-460.

3 Larsen EC, Christiansen OB, Kolte AM, Macklon N: New insights into mechanisms behind miscarriage. BMC Med 2013;11:154.

4 Zhang S, Lin H, Kong S, Wang S, Wang H, Wang H, Armant DR: Physiological and molecular determinants of embryo implantation. Mol Aspects Med 2013;34:939-980.

-5 Koot Y, Teklenburg G, Salker M, Brosens JJ, Macklon N: Molecular aspects of implantation failure. BBA-Mol Basis Dis 2012;1822:1943-1950.

-6 Timeva T, Shterev A, Kyurkchiev S: Recurrent Implantation Failure: The Role of the Endometrium. J Reprod Infertil 2014;15:173.

7 Kosova G, Stephenson MD, Lynch VJ, Ober C: Evolutionary forward genomics reveals novel insights into the genes and pathways dysregulated in recurrent early pregnancy loss. Hum Reprod 2015;30:519-529.

8 Krieg S, Fan X, Hong Y, Sang Q-X, Giaccia A, Westphal L, Lathi R, Krieg A, Nayak N: Global alteration in gene expression profiles of deciduas from women with idiopathic recurrent pregnancy loss. Mol Hum Reprod 2012;18:442-450.

-9 Wilcox AJ, Weinberg CR, O'Connor JF, Baird DD, Schlatterer JP, Canfield RE, Armstrong EG, Nisula BC: Incidence of early loss of pregnancy. New Engl J Med 1988;319:189-194.

10 Salker MS, Hosseinzadeh Z, Alowayed N, Zeng N, Umbach AT, Webster Z, Singh Y, Brosens JJ, Lang F: LEFTYA Activates the Epithelial $\mathrm{Na}+$ Channel (ENaC) in Endometrial Cells via Serum and Glucocorticoid Inducible Kinase SGK1. Cell Physiol \& Biochem Int J Exp Cell Physiol Biochem Pharmacol 2016;39:1295-1306.

-11 Salker MS, Steel JH, Hosseinzadeh Z, Nautiyal J, Webster Z, Singh Y, Brucker S, Lang F, Brosens JJ: Activation of SGK1 in Endometrial Epithelial Cells in Response to PI3K/AKT Inhibition Impairs Embryo Implantation. Cell Physiol Biochem 2016;39:2077-2087. 


\section{Cellular Physiology Cell Physiol Biochem 2017;41:501-509

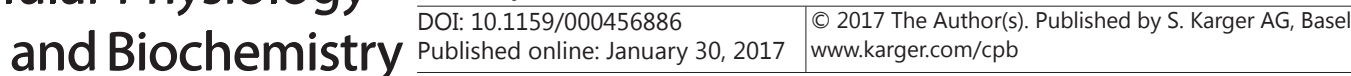 \\ Wang et al.: FABP4 and Embryonic Implantation}

12 Zimmerman A, Veerkamp J: New insights into the structure and function of fatty acid-binding proteins. Cell Mol Life Sci 2002;59:1096-1116.

13 Kralisch S, Fasshauer M: Adipocyte fatty acid binding protein: a novel adipokine involved in the pathogenesis of metabolic and vascular disease? Diabetologia 2013;56:10-21.

14 Tian Z, Zhao Z-A, Liang X-H, Zhang X-H, Sha A-G, Zhang Z-R, Yu Y-S, Yang Z-M: Expression and function of fatty acid-binding protein 4 during mouse decidualization. Fertil Steril 2011;95:2749-2752. e2745.

15 Zhu Q, Jin Y, Wang P, Wang H, Lu B, Wang Z, Dong M: Expression and function of fatty acid-binding protein 4 in epithelial cell of uterine endometrium. Cell Biol Int 2015;39:540-547.

- 16 Manohar M, Khan H, Sirohi VK, Das V, Agarwal A, Pandey A, Siddiqui WA, Dwivedi A: Alteration in endometrial proteins during early-and mid-secretory phases of the cycle in women with unexplained infertility. Plos One 2014;9:e111687.

17 Daikoku T, Cha J, Sun X, Tranguch S, Xie H, Fujita T, Hirota Y, Lydon J, DeMayo F, Maxson R: Conditional deletion of Msx homeobox genes in the uterus inhibits blastocyst implantation by altering uterine receptivity. Dev Cell 2011;21:1014-1025.

18 Nallasamy S, Li Q, Bagchi MK, Bagchi IC: Msx homeobox genes critically regulate embryo implantation by controlling paracrine signaling between uterine stroma and epithelium. PLos Genet 2012;8:e1002500.

19 Karaer A, Cigremis Y, Celik E, Gonullu RU: Prokineticin 1 and leukemia inhibitory factor mRNA expression in the endometrium of women with idiopathic recurrent pregnancy loss. Fertil Steril 2014;102:1091-1095.

20 Nair R, Khanna A, Singh K: Role of inflammatory proteins S100A8 and S100A9 in pathophysiology of recurrent early pregnancy loss. Placenta 2013;34:824-827.

21 Eskicioglu F, Laçin S, Ozbilgin K, Köse C: The role of selectins in the first trimester pregnancy loss. Ginekol Pol 2014;85:287-293.

22 Perfetto COH, Fan X, Dahl S, Krieg S, Westphal LM, Lathi RB, Nayak NR: Expression of interleukin-22 in decidua of patients with early pregnancy and unexplained recurrent pregnancy loss. J Assist Reprod Genet 2015;32:977-984.

23 Filant J, Spencer TE: Uterine glands: biological roles in conceptus implantation, uterine receptivity, and decidualization. Int J Dev Biol 2014;58:107. 